\title{
A systematic review of influences on implementation of peer support work for adults with mental health problems
}

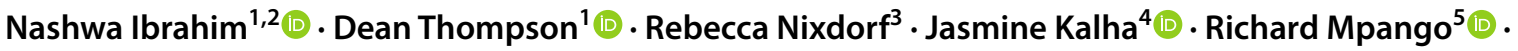

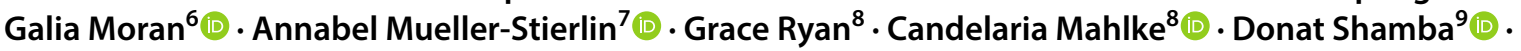 \\ Bernd Puschner $^{5}$ (D) Julie Repper ${ }^{10} \cdot$ Mike Slade $^{1}$ (D)
}

Received: 8 March 2019 / Accepted: 3 June 2019 / Published online: 8 June 2019

(c) The Author(s) 2019

\begin{abstract}
Purpose The evidence base for peer support work in mental health is established, yet implementation remains a challenge. The aim of this systematic review was to identify influences which facilitate or are barriers to implementation of mental health peer support work.

Methods Data sources comprised online databases $(n=11)$, journal table of contents $(n=2)$, conference proceedings $(n=18)$, peer support websites $(n=2)$, expert consultation $(n=38)$ and forward and backward citation tracking. Publications were included if they reported on implementation facilitators or barriers for formal face-to-face peer support work with adults with a mental health problem, and were available in English, French, German, Hebrew, Luganda, Spanish or Swahili. Data were analysed using narrative synthesis. A six-site international survey [Germany (2 sites), India, Israel, Tanzania, Uganda] using a measure based on the strongest influences was conducted. The review protocol was pre-registered (Prospero: CRD42018094838).

Results The search strategy identified 5813 publications, of which 53 were included. Fourteen implementation influences were identified, notably organisational culture (reported by $53 \%$ of papers), training (42\%) and role definition (40\%). Ratings on a measure using these influences demonstrated preliminary evidence for the convergent and discriminant validity of the identified influences.

Conclusion The identified influences provide a guide to implementation of peer support. For services developing a peer support service, organisational culture including role support (training, role clarity, resourcing and access to a peer network) and staff attitudes need to be considered. The identified influences provide a theory base to prepare research sites for implementing peer support worker interventions.
\end{abstract}

Keywords Peer support $\cdot$ Consumer-provider $\cdot$ Mental health $\cdot$ Systematic review $\cdot$ Implementation

\section{Introduction}

Peer support involves people with lived experience of mental health problems supporting others in their recovery from mental health problems [1-3]. Naturally occurring, informal

Nashwa Ibrahim and Dean Thompson are joint first authors.

Electronic supplementary material The online version of this article (https://doi.org/10.1007/s00127-019-01739-1) contains supplementary material, which is available to authorized users.

Mike Slade

m.slade@nottingham.ac.uk

Extended author information available on the last page of the article peer support has a very long history [4]. Examples of formal or intentional peer support, in which people with lived experience of mental health problems are employed as peer support workers (PSWs) in mental health services to support others, also extend back as far as the eighteenth century France and the moral treatment era [5]. Peer support promotes person-centred recovery by enabling contact between people with lived experience to foster a sense of connectedness by communicating shared experiences [6-8].

Peer support is increasingly being adopted around the world, as an approach to transforming mental health towards a recovery orientation [9]. A focus on recovery involves institutional transformation [10], with more emphasis on shared decision making [11] especially in relation to 
medication [12], and different approaches to compulsory care [13]. As part of this transformation, some countries such as Australia [14] and the UK [15] now mandate statutory mental health services to involve patients in service development and delivery, and there is increasing uptake of peer support around the world [16, 17]. One role expectation on PSWs is that they be carriers of a recovery culture into mental health systems [18], so introducing PSW roles into services is often challenging [19].

Peer support may not become properly embedded in routine clinical practice if stakeholders are unwilling to integrate it into existing practice or unable to make sense of the information required to operationalise it effectively [20]. Yet, there remain concerns about the organisation and implementation of peer support, with some professionals describing peer support colleagues as increasing workload [21, 22]. A non-systematic review of the barriers to PSW implementation identified a range of challenges, including cultural impediments, poor organisational arrangements, and inadequate overarching mental health policies [23]. Influences which facilitate implementation have not been systematically reviewed. Characterising the barriers and facilitators to PSW implementation would both support the development and rollout at scale of this new role and contribute to the organisational transformation involved in re-orienting services around recovery.

The aim of this systematic review was to identify facilitators and barriers to implementation of formal mental health peer support work.

\section{Method}

The review was conducted in the context of the Using Peer Support In Developing Empowering Mental Health Services (UPSIDES) Study, which is a multi-national research study using the Consolidated Framework for Implementation Research [24] to investigate PSW implementation in lowresource setting [25].

\section{Protocol and registration}

The protocol of this systematic review was developed in accordance with PRISMA guidelines [26] and registered on PROSPERO (International Prospective Register of Systematic Reviews) on 24 July 2018: CRD42018094838.

\section{Eligibility criteria}

Inclusion criteria were: (participants and intervention) studies about PSWs supporting adults 18 aged years or older with a primary diagnosis of mental illness; (outcome) identifies implementation facilitators or barriers for PSW implementation; (study design) randomised controlled trials, controlled before and after studies, cohort studies, case-control studies, cross-sectional studies and qualitative studies. Publications were included if reported in English, French, German, Hebrew, Luganda, Spanish or Swahili (chosen as languages in UPSIDES Study sites), with a date of publication on or before July 2018. Exclusion criteria were: substance misuse or addiction populations; intervention was mutual aid, peer-run organisations, naturally occurring peer support, peer navigation interventions and peer support delivered exclusively online. No studies were excluded on the basis of comparators, control conditions or setting.

\section{Information sources}

Six data sources were used: (1) the electronic bibliographic databases $(n=11)$ searched were MEDLINE (OVID), EMBASE (OVID), Cumulative Index of Nursing and Allied Health Literature (CINAHL) (EBSCHO), PsycINFO (OVID), Scopus, Web of Science, Google Scholar, OpenGrey, ProQuest Dissertations \& Theses A\&I, African Journals OnLine (AJOL) and Scientific Electronic Library Online (SciELO); (2) table of contents of Psychiatric Services and Psychiatric Rehabilitation Journal (chosen as publishers of PSW studies); (3) conference proceedings of European Network for Mental Health Service Evaluation (ENMESH) $(n=14,1994-2017)$ and Refocus on Recovery $(n=4,2010-2017)$ (chosen as recovery-relevant academic conferences with available proceedings); (4) websites (peersforprogress.org; cpr.bu.edu) (chosen as they host PSW materials); (5) a preliminary list of included publications was sent to experts $(n=38)$ requesting additional eligible publications; (6) forward citation tracking on all included records using Scopus and backward citation tracking through a hand-search of reference lists of included publications.

\section{Search strategy}

The search strategy was adapted from a published systematic review concerning peer support for people based on statutory mental health services [27]. The search strategy was modified for each database, and an example of the search strategy used for MedLine is shown in Online Resource 1. All searches were conducted from inception until July 2018.

\section{Study selection}

After removing duplicates, the titles and abstracts of all identified citations were screened for relevance against the inclusion criteria by DT, with a randomly selected 5\% sample independently assessed by RN. Concordance between the two reviewers was $93 \%$. Full texts were single screened by DT. Data extraction was then conducted on $10 \%$ of the 
included publications independently by DT and RN, who discussed their data extraction to check for adequate agreement. Date extraction for the remaining $90 \%$ of included publications was then conducted.

\section{Data abstraction}

For each included publication, information was extracted on (1) study participant inclusion and exclusion criteria, (2) a summary of the peer support intervention, (3) a summary of the characteristics of the PSW, (4) where the intervention was carried out including country and service setting and (5) factors that influenced implementation, whether the factor enabled or hindered implementation and the evidence that the stated implementation factor influenced implementation.

\section{Quality assessment}

The Critical Appraisal Skills Programme (CASP) was used to assess the quality of eligible studies. CASP checklists do not provide an overall scoring, so a scoring system used in a previous systematic review [28] was applied. Each CASP item rated 'yes' scored 1 point and each item rated 'no' scored 0 points. The percentage score for the 10-item CASP randomised controlled trial checklist, the 10-item CASP qualitative checklist, the 12-item CASP cohort checklist and the 11-item CASP case control checklist was calculated, with studies scoring $\geq 60 \%$ graded as good quality, studies scoring $45-59 \%$ graded as fair quality and studies scoring below $45 \%$ graded as poor [29, 30].

\section{Analysis}

A three-stage modified narrative synthesis [31] was conducted on the included papers. In stage 1 (developing a preliminary synthesis), facilitators and barriers to implementation of peer support work identified in included studies were synthesised. Findings were tabulated and an initial coding framework was developed through thematic analysis to group-related facilitators and barriers within overarching themes, called influences. Vote counting of the number of papers identifying each theme was performed to establish the strength of the theme. A preliminary draft of the implementation framework was developed and refined by analysts. In stage 2 (exploring relationships between studies), the implementation influences were compared for studies using a group-based modality (defined as one or more PSWs meeting several service users in a group) versus individual modality (defined as a PSW meeting a service user individually). Stage 3 (assessing the robustness of the synthesis) used two approaches. First, the findings from sub-group analysis of higher quality studies (rated as good) were compared with the framework from all included studies. Second, the identified influences coded in at least $10 \%$ of papers were adapted into a measure of PSW implementation readiness. The resulting Implementation Measure, shown in Online Resource 5, comprised eight items, each rated on a fourpoint scale: 0 (few or no facilitators present, many or all barriers present), 1 (more barriers than facilitators present), 2 (more facilitators than barriers present) or 3 (many or all facilitators present, few or no barriers present). The sum score ranges from 0 (implementation most difficult) to 24 . The measure was completed by the site lead in each of the six sites participating in the UPSIDES Study: Ulm (Germany), Hamburg (Germany), Kampala (Uganda), Dar es Salaam (Tanzania), Beer Sheva (Israel) and Pune (India). The sites include low-income (Kampala, Dar es Salaam), lower-middle (Pune) and high-income sites (Ulm, Hamburg, Beer Sheva), and sites with (Hamburg, Kampala, Beer Sheva, Pune) and without (Ulm, Dar es Salaam) experience of implementing peer support work. The goal was to provide preliminary evidence that the synthesis identified influences specific to peer support work (convergent validity) and not to generic influences such as resource level (discriminant validity).

\section{Results}

\section{Included studies}

The search identified 5813 publications, from which 53 met inclusion criteria. The flow diagram is shown in Fig. 1 and the complete data abstraction table including all references is shown in Online Resource 2.

The 53 included publications were conducted exclusively in high-income countries, mainly the USA $(n=30)$, UK $(n=7)$, Australia $(n=5)$, Canada $(n=3)$ and Republic of Ireland $(n=2)$, with single studies in Belgium, Germany, Hong Kong, Japan, The Netherlands and one two-country study from Israel and the USA. Designs comprised qualitative studies $(n=38)$, randomised controlled trials $(n=10)$, cohort studies $(n=4)$ and control studies $(n=1)$. Most included publications did not specify sample size for either PSWs or target clinical population. Studies were rated as good quality $(n=47)$, fair quality $(n=1)$ and poor quality $(n=5)$.

\section{Stage 1 (developing a preliminary synthesis)}

The influences identified in all included studies were tabulated and thematically analysed. The full synthesis, including all papers coded to each influence and the strength-oftheme data for each influence, is shown in Online Resource 3. Twelve types of influence, each comprising a set of facilitators and barriers, were identified: organisational 


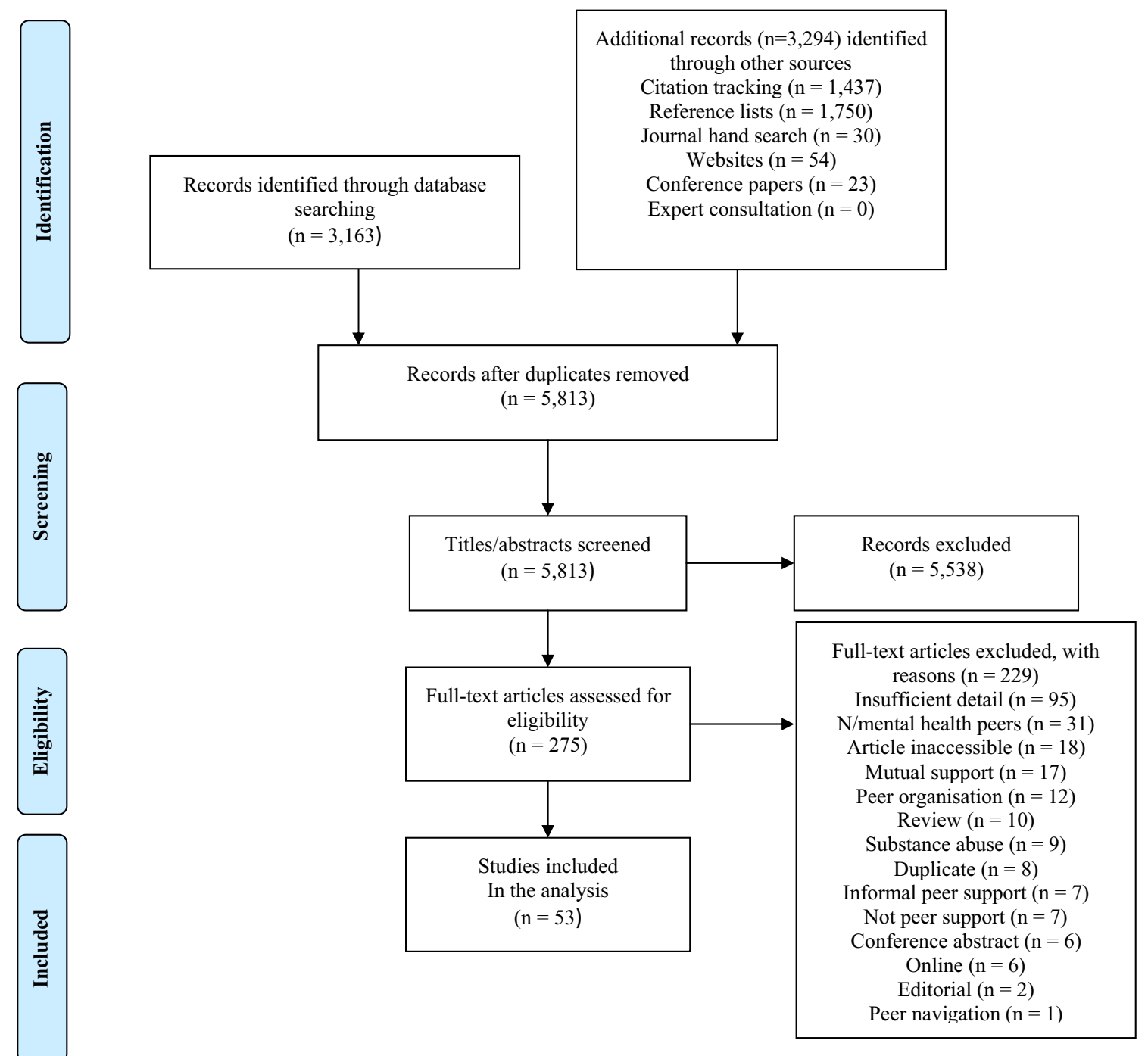

Fig. 1 Flow diagram of the study selection process

culture (coded in 53\% of papers); PSW training (42\%); PSW role definition (40\%); staff willingness and ability to work with PSWs (34\%); resource availability (21\%); financial arrangements (15\%); support for PSW well-being (13\%); PSWs access to a peer network (13\%); PSW ability to self-manage their own well-being (9\%); expectations held by PSWs (6\%); organisational processes (6\%); and research design needs $(2 \%)$. The facilitators and barriers for the eight most coded influences are shown in Table 1.

Additionally, two influences were identified for which direction was unclear or the evidence for whether they were facilitators or barriers was conflicting: match between PSW and patient (4\%); and emotional intensity in the work $(2 \%)$.

\section{Stage 2 (exploring relationships among studies)}

For the 42 (79\%) included studies which stated mode of implementation, 13 (30.9\%) were individual, 6 (14. 3\%) were group based, and 23 (54.8\%) were both individual and group based. Including only the 13 individual PSW studies in the thematic analysis did not lead to deletion of any of the eight strongest themes. However, the ordering changed, with the three strongest themes being 'organisational culture', 'PSW role definition' and 'resource availability'. Including only the six group-based PSW studies in the thematic analysis led to deletion of 'PSW role definition' and 'support for PSW well being' from the 
Table 1 Facilitators and barriers to implementation of peer support work

Implementation influence
\#publications identifying this influence
1. Organisational culture

1. Organisational culture
$n=28$
2. PSW training

$n=22$

3. PSW role definition

$n=21$

4. Staff willingness and ability to work with PSWs $n=18$

\section{Resource availability \\ $n=11$}

6. Financial arrangements
Description

\section{Facilitators}

The service has clear goals and a recovery orientation, with a culture of reflexive practice and an openness to change. PSWs occupy a central position within the service network. There is adequate communication with them, efforts are made to make cultural modifications for them, and they are seen by other staff as 'fitting in'. PSWs may be independent/ autonomous from mental health service (i.e. not managed within the organisation). Disability and work performance assessment are separated

\section{Barriers}

There is a risk-averse culture focussed on traditional clinical risk and peer-patient risk, with a lack of recovery orientation and coproduction. Treatment protocols are inflexible and conflict with person-centred principles. Traditional workplace hierarchies exist, and there is a lack of organisational commitment to PSWs. PSWs perceive that stigma exists about the PSW role, and PSWs are seen as outsiders. Co-optation occurs by incorporating PSWs into medical ways of working, leading to identity conflict between being a patient and being a worker

\section{Facilitators}

Training and supervision are available. There is an emphasis on skill building, with ongoing training and shadowing. PSWs are knowledgeable and skilled. Role practice and a transition period to working environment are supported

Barriers

There is a lack of supervision, or supervision challenges exist. Training is not reflective of PSW practice, or has a fixed structure. Training is inaccessible or non-existent. PSWs do not feel ready or have a sense of competence or confidence

\section{Facilitators}

The PSW role is clearly defined, and staff understand the PSW role. There is a code of conduct and training about appropriate boundaries, and adjustments are made to maintain a high role status for PSWs. There is a mandatory requirement for PSWs to share patient information with services, and the PSW understands patient confidentiality

Barriers

There is role ambiguity and uncertainty regarding the level of self-disclosure and boundaries. There is a mismatch between staff and PSW role expectations

\section{Facilitators}

PSWs receive a positive response from staff, including acceptance and trusting relationships. There is support from leadership. Staff have knowledge of the mandate to hire PSWs and receive advice on integrating PSWs

Barriers

There is a lack of staff understanding of PSWs, and staff hold reservations about hiring PSWs. Staff are uncertain or inexperienced on how to interact with PSWs, and PSWs perceive a lack of staff trust. There are conflicting time expectations between peers and staff-e.g. activities are scheduled when PSW is absent. There is a lack of contact between PSWs and staff. Staff hold fears about, or express hostility towards, PSWs. There is discrimination towards PSWs by staff. There is conflict between PSWs and staff, and a lack of respect towards them. For staff, there are disruptions to usual work patterns, and traditional power dynamics emerge

\section{Facilitators}

PSWs have access to the service resources they need, such as computer and Internet access, and there is open dialogue between PSWs and the service to ensure adequate support. PSWs have access to clinical records of patients they work with

\section{Barriers}

Information about peer support work is inaccessible to PSWs. PSWs have limited resources. PSWs do not have enough time to spend with patients. PSWs do not have authority over resources

\section{Facilitators}

There is enough money for PSWs to perform responsibilities. PSWs are paid for their work Barriers

There is a lack of funding for the PSW role, which has no or limited remuneration. Underemployment (not enough hours of work offered to PSW) is common. There is a lack of senior management support for funding 
Table 1 (continued)

\begin{tabular}{|c|c|}
\hline Implementation influence & Description \\
\hline $\begin{array}{l}\text { 7. Support for PSW well-being } \\
n=7\end{array}$ & $\begin{array}{l}\text { Facilitators } \\
\text { PSWs are taught self-care skills and helped to identify their own triggers of distress. There } \\
\text { is regular mental health screening for PSWs, and well-being procedures are in place for } \\
\text { them. Reasonable adjustments are made, e.g. sickness policy, holiday/annual leave, flex- } \\
\text { ible work schedule and reduced workload. Respect between peers is expected } \\
\text { Barriers } \\
\text { There is a stressful work milieu, and PSWs have an excessive workload }\end{array}$ \\
\hline $\begin{array}{l}\text { 8. PSW access to a peer network } \\
n=7\end{array}$ & $\begin{array}{l}\text { Facilitators } \\
\text { PSWs have contact with a peer network outside of the mental health organisation in which } \\
\text { the PSW is based } \\
\text { Barriers } \\
\text { PSWs have difficulty in forming peer relationships or lose personal peer networks }\end{array}$ \\
\hline
\end{tabular}

framework. The ordering did not markedly change, with 'organisational culture' remaining the strongest theme.

\section{Stage 3 (assessing the robustness of the synthesis)}

The quality rating for studies is shown in Online Resource 4. Excluding the five studies rated as poor quality and the one study rated as fair quality did not influence the content or strength-of-theme ordering of the narrative synthesis.

The Implementation Measure, shown in Online Resource 5 , was based on the most coded influences, shown in Table 1. The resulting eight-item measure (range 0-3 for each item) was rated at each site, and the results are shown in Table 2.

No significant floor or ceiling effects were identified: the mean rating per item ranged from 1.2 to 1.7 (scale midpoint 1.5) and each item except item 6 had at least a twopoint range in scores. The measure successfully differentiated between sites with a history of PSW implementation (Kampala, Beer Sheva, Hamburg, Pune; mean item score 1.8) and sites without such a history (Dar es Salaam, Ulm; mean score 0.8 ), providing preliminary evidence of convergent validity. The similar scores for low-income and lower-middle income sites (Dar es Salaam, Kampala, Pune; mean score 1.3) and high-income sites (Beer Sheva, Hamburg, Ulm; mean score 1.5) provide preliminary evidence of discriminant validity.

\section{Discussion}

This systematic review and narrative synthesis has identified facilitators and barriers to the implementation of formal peer support work in mental health services. Fourteen types of influence were identified, and scores from a measure based on the most identified eight influences provides preliminary evidence that these influences are specific to peer support work implementation rather than relating to general resource availability.

This review has developed a determinant framework [32], in which facilitators and barriers (determinants) of the implementation of formal peer support work in mental health were identified. Determinants may operate as effect modifiers, mediators or may form links in a chain of causal mechanism [33]. To illustrate, successful PSW implementation may only

Table 2 Implementation Measure scores at sites $(n=6)$ varying on income level and PSW implementation experience

\begin{tabular}{|c|c|c|c|c|c|c|c|}
\hline & $\begin{array}{l}\text { Ulm } \\
\text { Germany }\end{array}$ & $\begin{array}{l}\text { Hamburg } \\
\text { Germany }\end{array}$ & $\begin{array}{l}\text { Kampala } \\
\text { Uganda }\end{array}$ & $\begin{array}{l}\text { Dar es Salaam } \\
\text { Tanzania }\end{array}$ & $\begin{array}{l}\text { Beer Sheva } \\
\text { Israel }\end{array}$ & $\begin{array}{l}\text { Pune } \\
\text { India }\end{array}$ & Mean \\
\hline Income level & High & High & Low & Low & High & Lower-middle & \\
\hline PSW experience & No & Yes & Yes & No & Yes & Yes & \\
\hline 1. Organisational culture & 0 & 1 & 2 & 1 & 2 & 1 & 1.2 \\
\hline 2. PSW training & 0 & 3 & 2 & 1 & 2 & 1 & 1.5 \\
\hline 3. PSW role definition & 0 & 2 & 2 & 2 & 1 & 1 & 1.3 \\
\hline $\begin{array}{l}\text { 4. Staff willingness and ability to } \\
\text { work with PSWs }\end{array}$ & 0 & 1 & 2 & 2 & 3 & 0 & 1.3 \\
\hline 5. Resource availability & 0 & 2 & 2 & 1 & 3 & 0 & 1.3 \\
\hline 6. Financial arrangements & 2 & 1 & 1 & 1 & 3 & 2 & 1.7 \\
\hline 7. Support for PSW well-being & 1 & 2 & 2 & 2 & 2 & 1 & 1.7 \\
\hline 8. PSW access to a peer network & 0 & 3 & 2 & 0 & 3 & 1 & 1.5 \\
\hline Total & 3 & 15 & 15 & 10 & 19 & 7 & 15 \\
\hline
\end{tabular}


be possible in services which place value on lived experience as a resource benefiting others [19], but the presence of PSWs in the service supports that change by reducing in-system stigma [34] and 'them-and-us' distinctions [35]. So in addition to the direct effectiveness of PSW as an intervention [36], the presence of PSWs influences how other treatments and interventions are provided [37, 38].

The identified influences are consistent with implementation science frameworks. For example, they can be mapped onto the domains of the consolidated framework for implementation research (CFIR) [24], which is a widely used determinant framework to guide implementation [32], and the implementation framework being used in the UPSIDES Study. The CFIR inner setting domain encompasses organisational culture (influence 1 in Table 1), PSW training if provided internally (influence 2), PSW well-being (influence 7); and peer network access if provided internally (influence 8 ). The CFIR outer setting domain encompasses: role definition if externally defined (influence 3); resource availability (influence 5); financial arrangements (influence 6); and peer network access if provided externally (influence 8 ). The CFIR individuals domain encompasses staff willingness and ability (influence 4), and the CFIR implementation process domain encompasses PSW role definition if organisationally defined (influence 3) and PSW training (influence 2). Addressing these CFIR domains increases the likelihood of successful implementation [39].

\section{Strengths and limitations}

The strengths of this review include the systematic and multi-language search strategy, the robustness of the methodology including quality appraisal and multiple analysts, and preliminary validation of the narrative synthesis through a five-country survey. Several limitations can be identified. First, the exclusion criteria included online peer support studies, which may involve different types of implementation facilitators and barriers, such as technological access. Second, implementation expertise is often gained through experience, so the reliance in this review on published papers could have been supplemented with interviews with a purposive sample of experts. Third, the absence of any identified studies from low-resource settings may reflect the relative difficulty in accessing such reports. A stronger focus on grey literature, widening the inclusion criteria and a wider expert consultation, might have identified reports from lowerincome settings, e.g. China [40] and Uganda [41], or related studies such as the ReDeAmericas Program in Latin America (http://www.cugmhp.org/research/redeamericas). Fourth, data extraction was conducted by two analysts on only $10 \%$ of included publications, whereas double data extraction is recommended on $100 \%$ of included publications [42]. The possibility of publication bias and heterogeneity in included papers are also limitations. Fifth, included publications were not analysed by diagnosis of PSW or target population, since peer support is a transdiagnostic intervention [43]. Finally, whilst strength of theme was used to order the identified influences, the magnitude and mechanisms of each influence were not investigated. So whilst organisational culture was the most identified influence, it may be that other influences have a greater impact on implementation, or that sub-levels of organisations (e.g. team, service, leadership etc.) exert different types of influence on implementation.

\section{Implications for practice and research}

The identified influences provide a theoretical foundation to guide PSW implementation in new settings. The Implementation Measure was shown to be usable in both lowerand higher-income countries, and to differentiate between sites with and without PSW implementation experience. The Implementation Measure can therefore be provisionally recommended for two uses.

First, for clinical services intending to implement a new PSW service, it provides a synthesised set of influences to address as part of the service development. In addition to the substantial evidence about PSW effectiveness [27, 44], there is emerging economic evidence supporting PSW implementation [45] and preliminary evidence that PSW services create a social return on investment [46]. Implementation in new clinical services is therefore likely to increase. The Implementation Measure as well as more locally developed approaches, such as the Team Recovery Implementation Plans (TRIP) approach developed in England [47], identifies the key influences to target. The most identified influence was organisational culture, and this is an international challenge [48]. Evidence-based approaches are emerging to change organisational culture, including a greater emphasis on supporting strengths [49-51], self-management [52], hope [53,54], well-being [55-57] and more use of new interventions such as positive psychology [58, 59], recovery colleges [60-62] and a greater focus on human rights [63]. New measures to evaluate recovery-related outcomes are also becoming available [64, 65].

Second, the Implementation Measure can be used in research to inform the implementation strategy for manualised PSW interventions. This is the approach being taken in the UPSIDES Study, in which the quantitative Implementation Measure score and focus group data are being integrated to develop a detailed situation analysis, to inform the implementation approach to be used in each site [25]. Maximising the presence of facilitators and addressing barriers will support effective and sustainable PSW implementation in a range of settings.

Acknowledgements The study Using Peer Support In Developing Empowering Mental Health Services (UPSIDES) is a multicentre collaboration between the Department for Psychiatry and Psychotherapy 
II at Ulm University, Germany (Bernd Puschner, coordinator); the Institute of Mental Health at University of Nottingham, UK (Mike Slade); the Department of Psychiatry at University Hospital HamburgEppendorf, Germany (Candelaria Mahlke); Butabika National Referral Hospital, Uganda (David Basangwa); the Centre for Global Mental Health at London School of Hygiene and Tropical Medicine, UK (Grace Ryan); Ifakara Health Institute, Dar es Salaam, Tanzania (Donat Shamba); the Department of Social Work at Ben Gurion University of the Negev, Beer Sheva, Israel (Galia Moran); and the Centre for Mental Health Law and Policy, Pune, India (Jasmine Kalha). UPSIDES received funding from the European Union's Horizon 2020 Research and Innovation Programme under Grant Agreement No 779263. MS acknowledges the support of the Center for Mental Health and Substance Abuse, University of South-Eastern Norway and the NIHR Nottingham Biomedical Research Centre. This publication reflects only the authors' views. The Commission is not responsible for any use that may be made of the information it contains. The funding body had no role in the design of the study and in writing the manuscript.

\section{Compliance with ethical standards}

Conflict of interest On behalf of all authors, the corresponding author states that there is no conflict of interest.

Ethical standards The manuscript does not contain clinical studies or patient data.

Open Access This article is distributed under the terms of the Creative Commons Attribution 4.0 International License (http://creativeco mmons.org/licenses/by/4.0/), which permits unrestricted use, distribution, and reproduction in any medium, provided you give appropriate credit to the original author(s) and the source, provide a link to the Creative Commons license, and indicate if changes were made.

\section{References}

1. Repper J, Carter T (2011) A review of the literature on peer support in mental health services. J Mental Health 20:392-411

2. Davidson L et al (2012) Peer support among persons with severe mental illnesses: a review of evidence and experience. World Psychiatry 11:123-128

3. Puschner B (2018) Peer support and global mental health. Epidemiol Psychiatr Sci 27:413-414

4. Werner-Seidler A et al (2017) The relationship between social support networks and depression in the 2007 National Survey of Mental Health and Well-being. Soc Psychiatry Psychiatr Epidemiol 52:1463-1473

5. Pelletier JF, Davidson L (2015) At the very roots of psychiatry as a new medical specialty: the Pinel-Pussin partnership. Sante Mentale au Quebec 40:19-33

6. Steigman PJ et al (2014) Psychiatric symptoms moderate the effects of mental illness self-management in a randomized controlled trial. J Nerv Mental Dis 202:193-199

7. Leamy $\mathrm{M}$ et al (2011) A conceptual framework for personal recovery in mental health: systematic review and narrative synthesis. $\mathrm{Br}$ J Psychiatry 199:445-452

8. Bird V et al (2014) Fit for purpose? Validation of the conceptual framework of personal recovery with current mental health service users. Aust N Z J Psychiatry 48:644-653

9. World Health Organization (2013) Mental health action plan 20132020. WHO, Geneva
10. Slade M et al (2014) Uses and abuses of recovery: implementing recovery-oriented practices in mental health systems. World Psychiatry $13: 12-20$

11. Hamann J, Heres S (2014) Adapting shared decision making for individuals with severe mental illness. Psychiatr Serv 65:1483-1486

12. Deegan P, Drake R (2006) Shared decision making and medication management in the recovery process. Psychiatr Serv 57:1636-1639

13. Burns $\mathrm{T}$ et al (2013) Community treatment orders for patients with psychosis (OCTET): a randomised controlled trial. Lancet 381(9878): 1627-1633

14. Department of Health (2017) The fifth national mental health and suicide prevention plan. Commonwealth of Australia, Canberra

15. HM Government (2011) No health without mental health. Delivering better mental health outcomes for people of all ages. Department of Health, London

16. Moran GS (2018) The mental health consumer movement and peer providers in Israel. Epidemiol Psychiatr Sci 27:420-426

17. Pathare S, Kalha J, Krishnamoorthy S (2018) Peer support for mental illness in India: an underutilised resource. Epidemiol Psychiatr Sci 27:415-419

18. Carr S (2018) From psychiatric patient to citizen revisited AU. Disabil Soc 33:815-817

19. Gillard $S$ et al (2013) Introducing peer worker roles into UK mental health service teams: a qualitative analysis of the organisational benefits and challenges. BMC Health Serv Res 13:188

20. May C (2013) Towards a general theory of implementation. Implement Sci 8:18

21. Gillard S, Holley J (2014) Peer workers in mental health services: literature overview. Adv Psychiatr Treat 20:286-292

22. Collins R, Firth L, Shakespeare T (2016) "Very much evolving": a qualitative study of the views of psychiatrists about peer support workers. J Mental Health 25:278-283

23. Vandewalle J et al (2016) Peer workers' perceptions and experiences of barriers to implementation of peer worker roles in mental health services: a literature review. Int J Nurs Stud 60:234-250

24. Damschroder LJ et al (2009) Fostering implementation of health services research findings into practice: a consolidated framework for advancing implementation science. Implement Sci 4:50

25. Puschner B et al (2019) Using peer support in developing empowering mental health services (UPSIDES): background, rationale and methodology. Ann Glob Health 85(53):1-10

26. Moher D et al (2009) Preferred reporting items for systematic reviews and meta-analyses: the PRISMA statement. BMJ 339:332-336

27. Pitt V et al (2013) Consumer-providers of care for adult clients of statutory mental health services. Cochrane Database Syst Rev 3:CD004807

28. Matthews W et al (2018) Classification of tendon matrix change using ultrasound imaging: a systematic review and meta-analysis. Ultrasound Med Biol 44:2059-2080

29. Kennelly J (2011) Methodological approach to assessing the evidence. In: Handler A, Kennelly J, Peacock N (eds) Reducing racial/ ethnic disparities in reproductive and perinatal outcomes. Springer, Boston, pp 7-19

30. Adhia DB et al (2013) Validity and reliability of palpation-digitization for non-invasive kinematic measurement-a systematic review. Man Ther 18:26-34

31. Popay J et al (2006) Guidance on the conduct of narrative synthesis in systematic reviews. Prod ESRC Methods Programme Version 1:b92

32. Nilsen P (2015) Making sense of implementation theories, models and frameworks. Implement Sci 10:53

33. Flottorp SA et al (2013) A checklist for identifying determinants of practice: a systematic review and synthesis of frameworks and taxonomies of factors that prevent or enable improvements in healthcare professional practice. Implement Sci 8(1):35 
34. Henderson C et al (2014) Mental health-related stigma in health care and mental health-care settings. Lancet Psychiatry 1:467-482

35. Schrank B et al (2015) Staff views on wellbeing for themselves and for service users. J Mental Health 24:48-53

36. Johnson S et al (2018) Peer-supported self-management for people discharged from a mental health crisis team: a randomised controlled trial. Lancet 392:409-418

37. Meadows G et al (2019) REFOCUS-PULSAR recovery-oriented practice training in specialist mental health care: a steppedwedge cluster randomised controlled trial. Lancet Psychiatry 6:103-114

38. Slade M et al (2015) Supporting recovery in patients with psychosis using adult mental health teams (REFOCUS): a multi-site cluster randomised controlled trial. Lancet Psychiatry 2:503-514

39. Keith RE et al (2017) Using the consolidated framework for implementation research (CFIR) to produce actionable findings: a rapidcycle evaluation approach to improving implementation. Implement Sci 12:15

40. Fan Y et al (2018) A community-based peer support service for persons with severe mental illness in China. BMC Psychiatry 18:170

41. Hall C et al (2017) Brain Gain in Uganda: a case study of peer working as an adjunct to statutory mental health care in a low-income country. In: White R et al (eds) Palgrave handbook of sociocultural perspectives on global mental health. Palgrave Macmillan, London, pp 633-655

42. Higgins JP, Churchill R, Chandler J, Cumpston MS (eds) (2017) Cochrane handbook for systematic reviews of interventions version 5.2.0 (updated June 2017). The Cochrane Collaboration, Available from http://www.training.cochrane.org/handbook

43. Charles A et al A systematic review of modifications to peer support work for adults with mental health (in submission)

44. Lloyd-Evans B et al (2014) A systematic review and meta-analysis of randomised controlled trials of peer support for people with severe mental illness. BMC Psychiatry 14:39

45. Slade $M$ et al (2017) ImROC briefing paper 14. Recovery: the business case. ImROC, Nottingham

46. McCorriston E, Stevenson N (2018) SROI evaluative analysis: recovery college east peer education programme (PEP). Anglia Ruskin University, Cambridge

47. Repper J, Perkins R (2013) The team recovery implementation plan: a framework for creating recovery-focused services. Implementing Recovery Through Organisational Change, London

48. Slade M, Amering M, Oades L (2008) Recovery: an international perspective. Epidemiol Psychiatr Soc 17:128-137

49. Bird V et al (2012) Assessing the strengths of mental health service users—-systematic review. Psychol Assess 24:1024-1033
50. Tse S et al (2016) Uses of strength-based interventions for people with serious mental illness: a critical review. Int J Soc Psychiatry 62:281-291

51. Priebe $\mathrm{S}$ et al (2014) Resource-oriented therapeutic models in psychiatry-a conceptual review. Br J Psychiatry 204:256-261

52. Cook JA et al (2012) Results of a randomized controlled trial of mental illness self-management using Wellness Recovery Action Planning. Schizophr Bull 38:881-891

53. Schrank B et al (2012) Determinants, self-management strategies and interventions for hope in people with mental disorders: systematic search and narrative review. Soc Sci Med 74:554-564

54. Kirst $\mathrm{M}$ et al (2014) The promise of recovery: narratives of hope among homeless individuals with mental illness participating in a Housing First randomised controlled trial in Toronto, Canada. BMJ Open 4:e004379

55. Schrank B et al (2014) WELLFOCUS PPT-modified positive psychotherapy to improve well-being in psychosis: study protocol for a pilot randomised controlled trial. Trials 15(1):203

56. Schrank B et al (2014) A conceptual framework for improving wellbeing in people with a diagnosis of psychosis. Epidemiol Psychiatr Sci 23:377-387

57. Slade M, Oades L, Jarden A (eds) (2017) Wellbeing, recovery and mental health. Cambridge University Press, Cambridge

58. Slade M et al (2017) Positive psychotherapy for psychosis. Routledge, Hove

59. Slade $M$ (2010) Mental illness and well-being: the central importance of positive psychology and recovery approaches. BMC Health Serv Res 10:26

60. Crowther A, Taylor A, Toney R, Meddings S, Whale T, Jennings H, Pollock K, Bates P, Henderson C, Waring J, Slade M (2018) The impact of recovery colleges on mental health staff, services and society. Epidemiol Psychiatr Sci. https://doi.org/10.1017/S204579601 800063X

61. Toney R et al (2018) Mechanisms of action and outcomes for students in recovery colleges. Psychiatr Serv 69:1222-1229

62. Toney $\mathrm{R}$ et al (2018) Development and evaluation of a recovery college fidelity measure. Can J Psychiatry. https://doi.org/10.1177/07067 43718815893

63. Funk M, Drew N (2017) WHO Quality Rights: transforming mental health services. Lancet Psychiatry 4:826-827

64. Williams J et al (2012) Measures of the recovery orientation of mental health services: systematic review. Soc Psychiatry Psychiatr Epidemiol 47:1827-1835

65. Williams J et al (2015) Development and evaluation of the INSPIRE measure of staff support for personal recovery. Soc Psychiatry Psychiatr Epidemiol 50:777-786

\section{Affiliations}

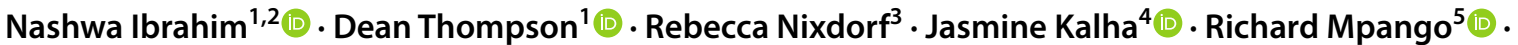

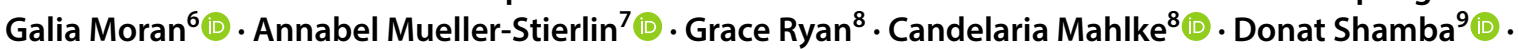 Bernd Puschner $^{5}$. Julie Repper ${ }^{10} \cdot$ Mike Slade $^{1}$ (])}

1 School of Health Sciences, Institute of Mental Health, University of Nottingham, Triumph Road, Nottingham NG7 2TU, UK

2 Psychiatric and Mental Health Nursing Department, Faculty of Nursing, Mansoura University, Mansoura, Egypt

3 Department of Psychiatry, Universitätsklinikum Hamburg-Eppendorf, Hamburg, Germany

4 Centre for Mental Health Law and Policy, Indian Law Society, Pune, India
5 Butabika National Referral Hospital, Kampala, Uganda

6 Ben Gurion University of the Negev, Beer Sheva, Israel

7 Department of Psychiatry II, Ulm University, Ulm, Germany

8 London School of Hygiene and Tropical Medicine, London, UK

9 Ifakara Health Institute, Ifakara, Tanzania

10 Nottinghamshire Healthcare NHS Foundation Trust, Nottingham, UK 